\title{
Transferring State Representations in Hierarchical Spiking Neural Networks
}

Barna Zajzon*, Renato Duarte and Abigail Morrison

10.07.2018 | IJCNN, SS7: ADVANCES IN RESERVOIR COMPUTING b.zajzon@fz-juelich.de 


\section{Introduction}

- Hierarchical modularity as a design principle

- Neocortex as a large distributed hierarchy of recurrent spiking networks

- Mechanism for information transfer

What features enable efficient information transfer? ... in a computationally useful way?

Context: Reservoir Computing

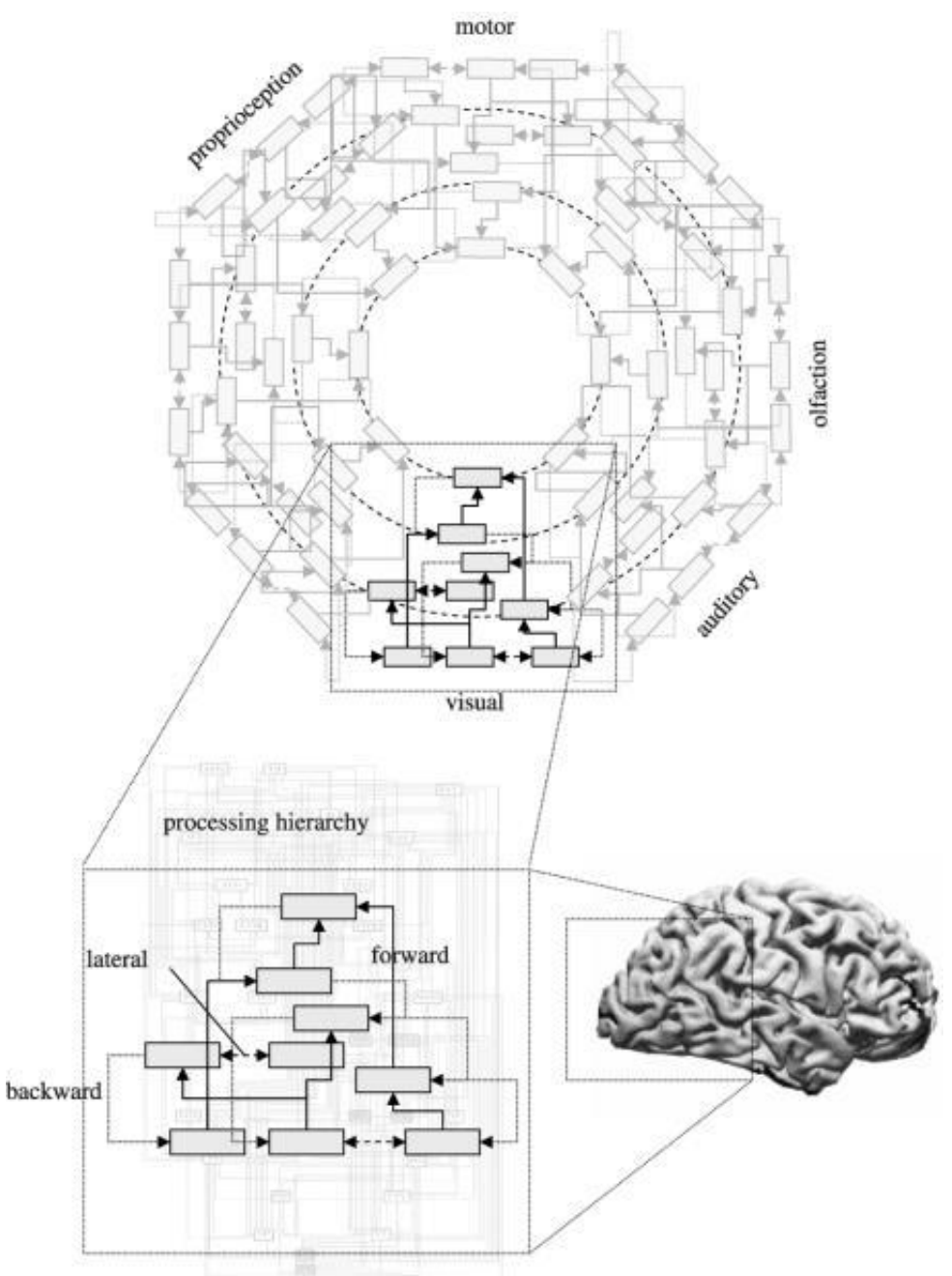

Friston, K. (2005)

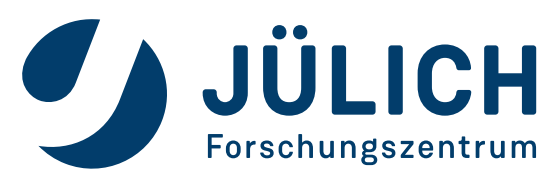




\section{Setup}

- 4 identical layers of balanced random networks

- 10000 spiking neurons (LIF)

- Static synaptic weights

random feed-forward

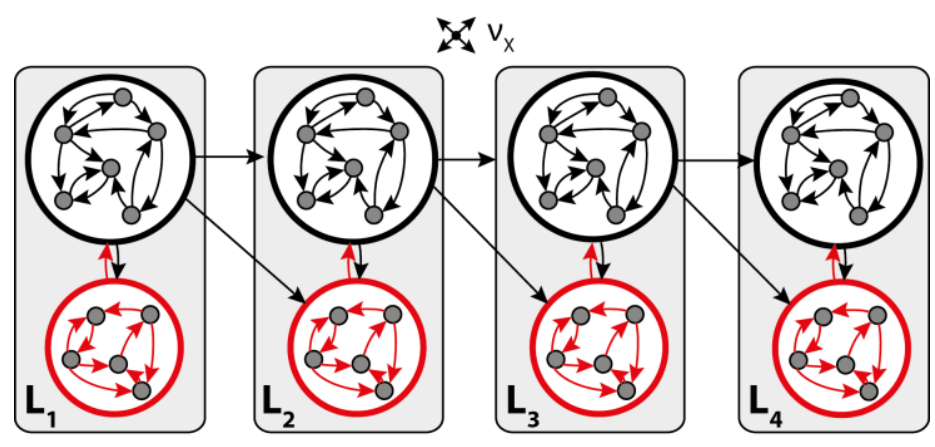

$>$ Each neuron $\rightarrow$ same amount of excitatory input

$>$ Tune parameters for asynchronous irregular activity (first 2 layers) 


\section{Setup}

- 4 identical layers

- 10000 spiking neurons (LIF)

- Static synaptic weights

- Poisson input to sub-populations in $\mathrm{L}_{1}$

random feed-forward

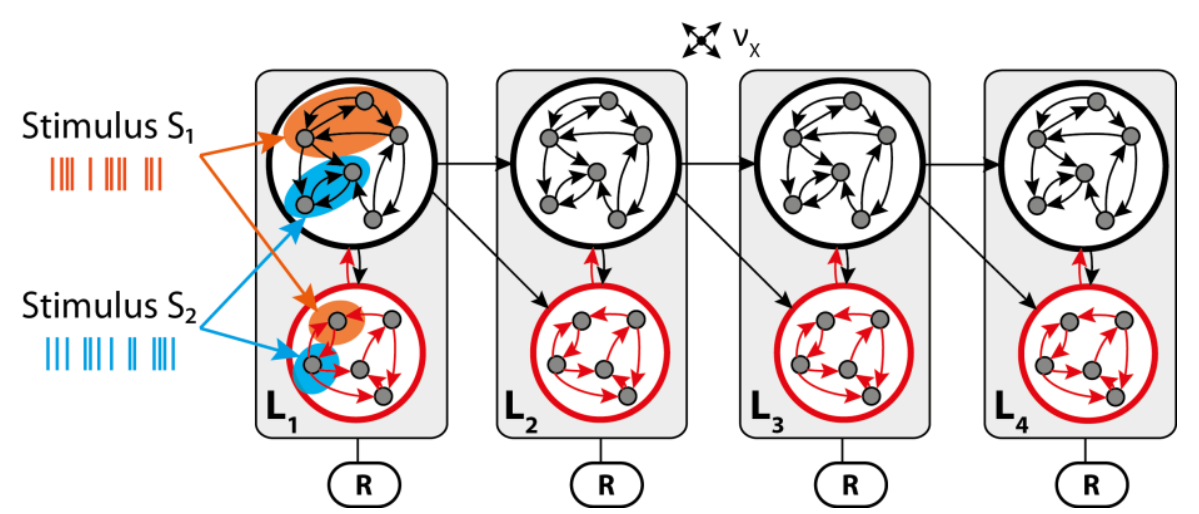

$>$ Each neuron $\rightarrow$ same amount of excitatory input

$>$ Tune parameters for asynchronous irregular activity (first 2 layers)

$>$ Readout from E populations in each layer

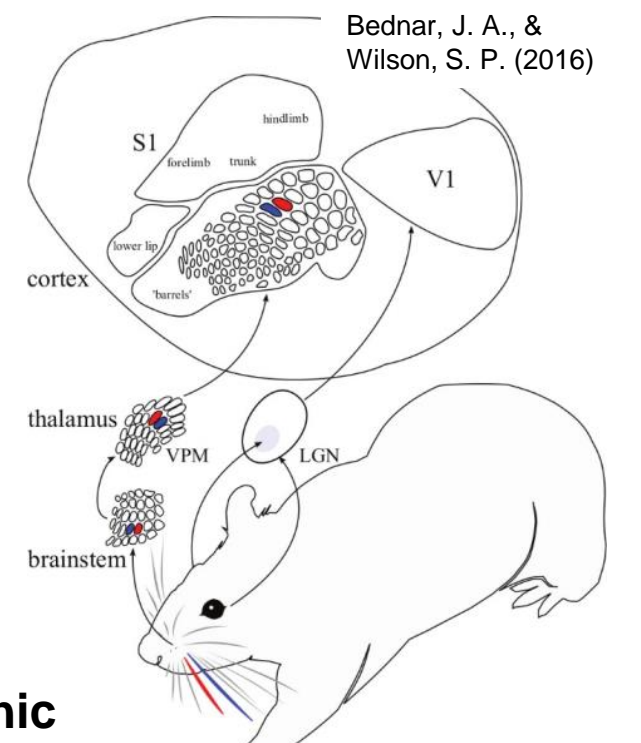

|||| | ||| ||

Stimulus $\mathrm{S}_{2}$ ||| ||| | || |||

\section{topographic}

$x v_{x}$ in

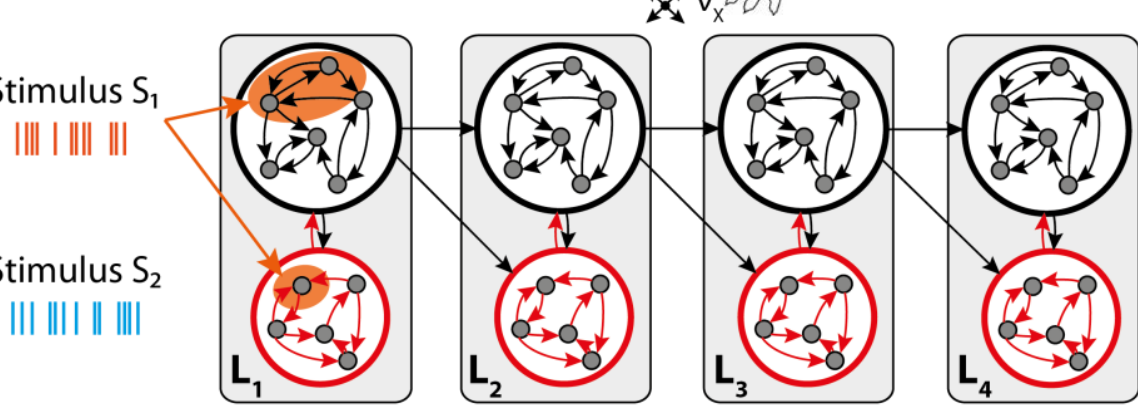




\section{Setup}

- 4 identical layers

- 10000 spiking neurons (LIF)

- Static synaptic weights

- Poisson input to sub-populations in $\mathrm{L}_{1}$

random feed-forward

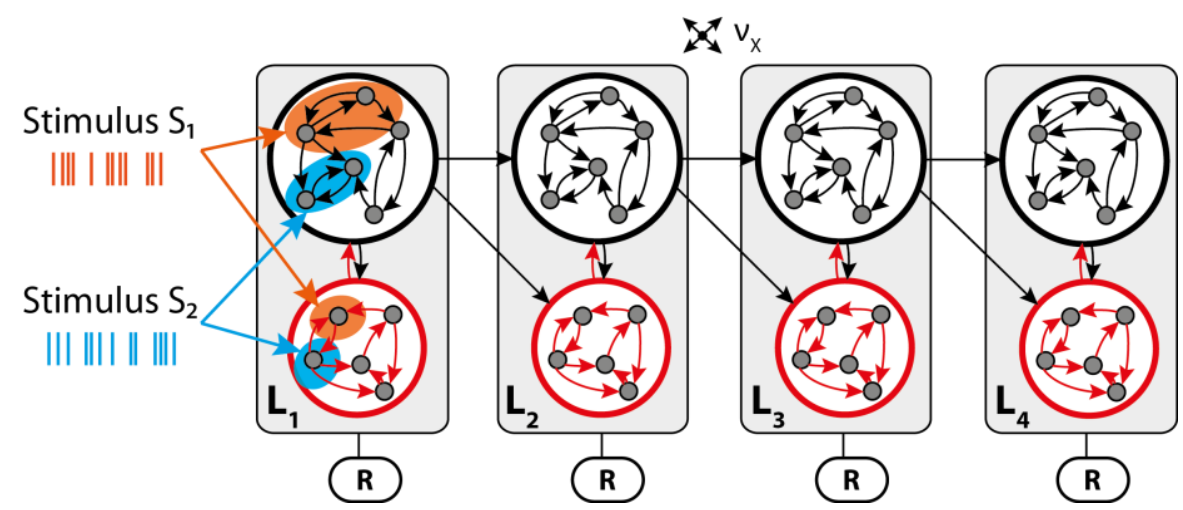

$>$ Each neuron $\rightarrow$ same amount of excitatory input

$>$ Tune parameters for asynchronous irregular activity (first 2 layers)

$>$ Readout from E populations in each layer

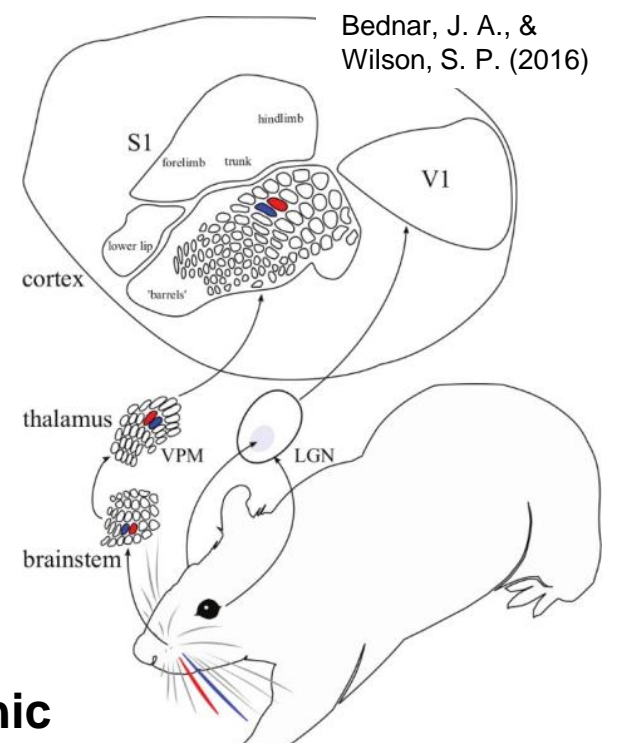

Stimulus $\mathrm{S}$

||||| | ||| ||

Stimulus $\mathrm{S}_{2}$

||| ||| || ||||

\section{topographic}

$x v_{x}=$

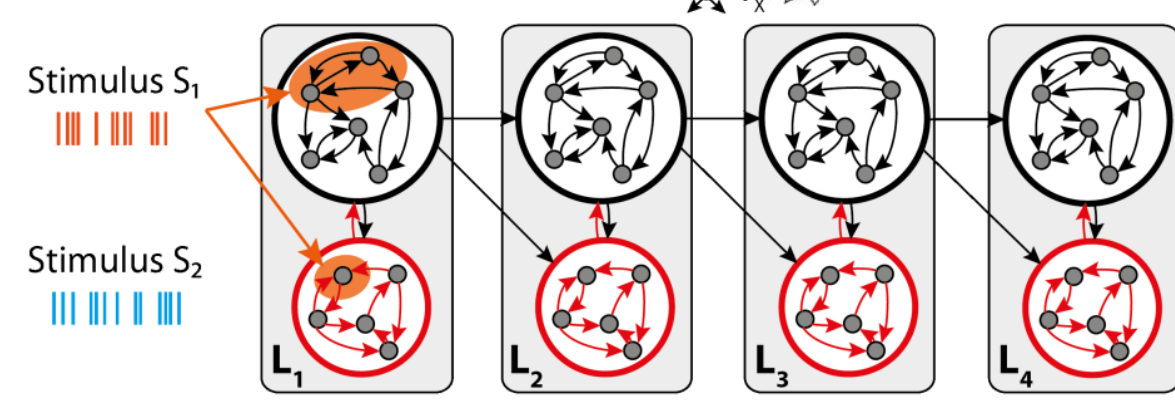

$>$ Stimulus-specific sub-populations conserved across the hierarchy through structured connectivity

$>$ Randomly selected, overlap allowed

$>$ Receptive field size fixed across the hierarchy

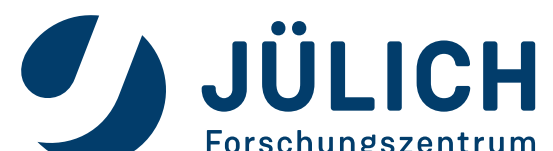




\section{Setup}

- 4 identical layers

- 10000 spiking neurons (LIF)

- Static synaptic weights

- Poisson input to sub-populations in $\mathrm{L}_{1}$

random feed-forward

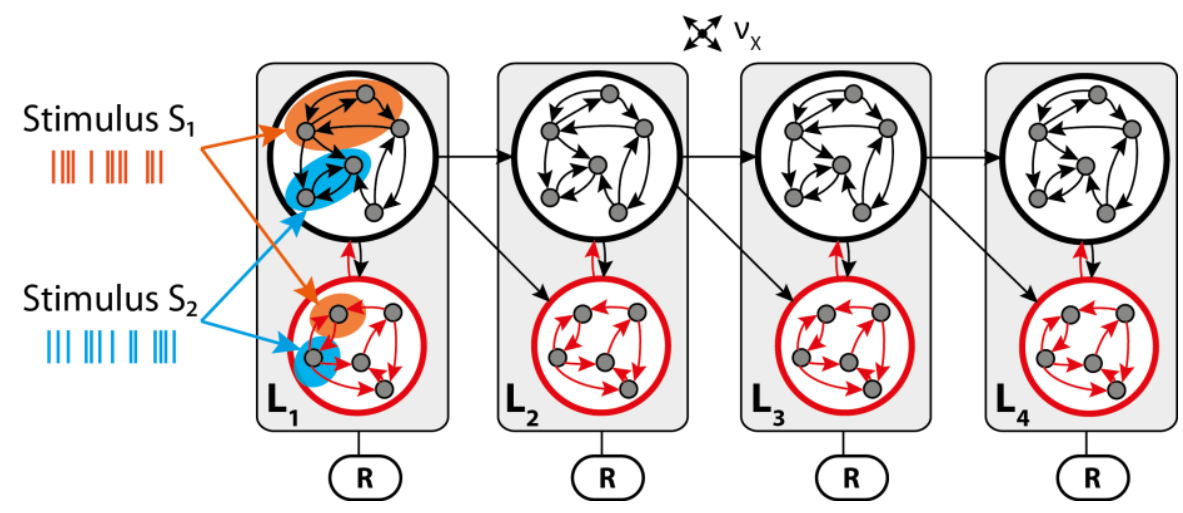

$>$ Each neuron $\rightarrow$ same amount of excitatory input

$>$ Tune parameters for asynchronous irregular activity (first 2 layers)

$>$ Readout from E populations in each layer

\section{topographic}

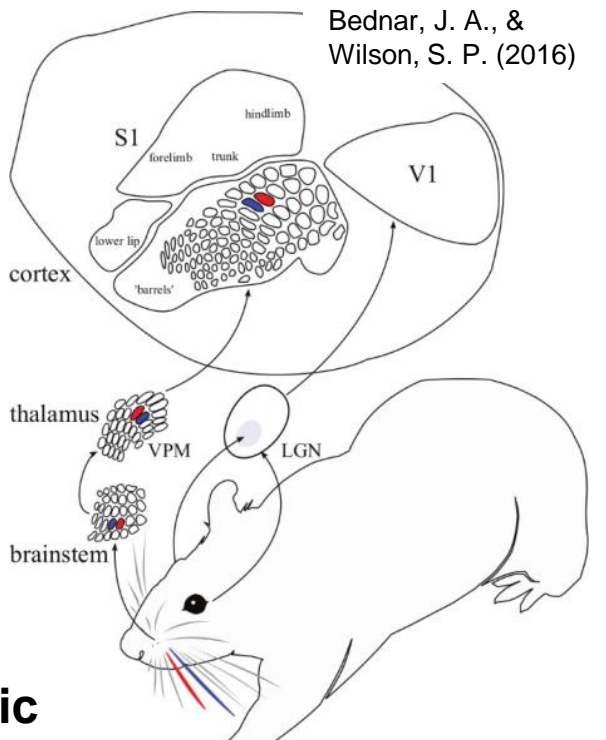

Stimulus $\mathrm{S}_{2}$ ||| ||| || |||

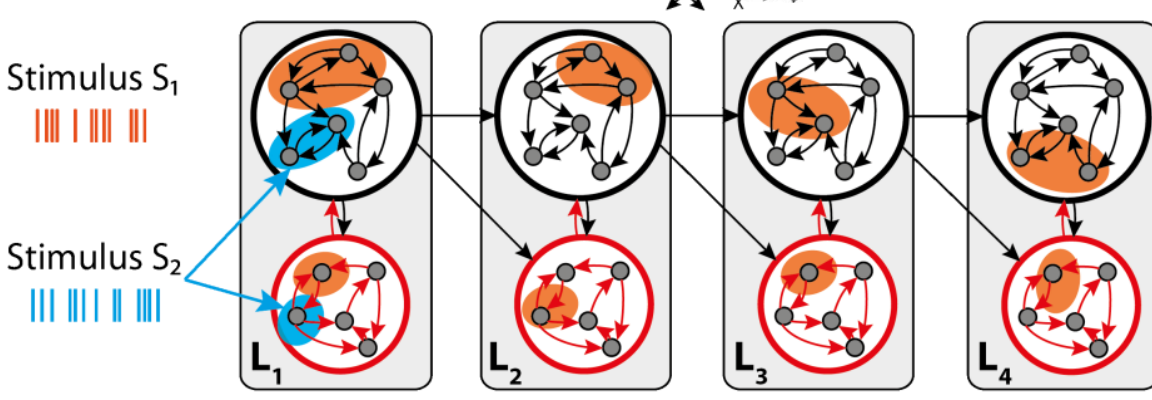

$x v_{x} \approx$

$>$ Stimulus-specific sub-populations conserved across the hierarchy through structured connectivity

$>$ Randomly selected, overlap allowed

$>$ Receptive field size fixed across the hierarchy

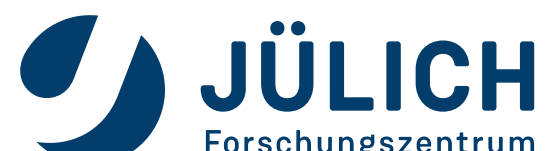




\section{Setup}

- 4 identical layers

- 10000 spiking neurons (LIF)

- Static synaptic weights

- Poisson input to sub-populations in $\mathrm{L}_{1}$

random feed-forward

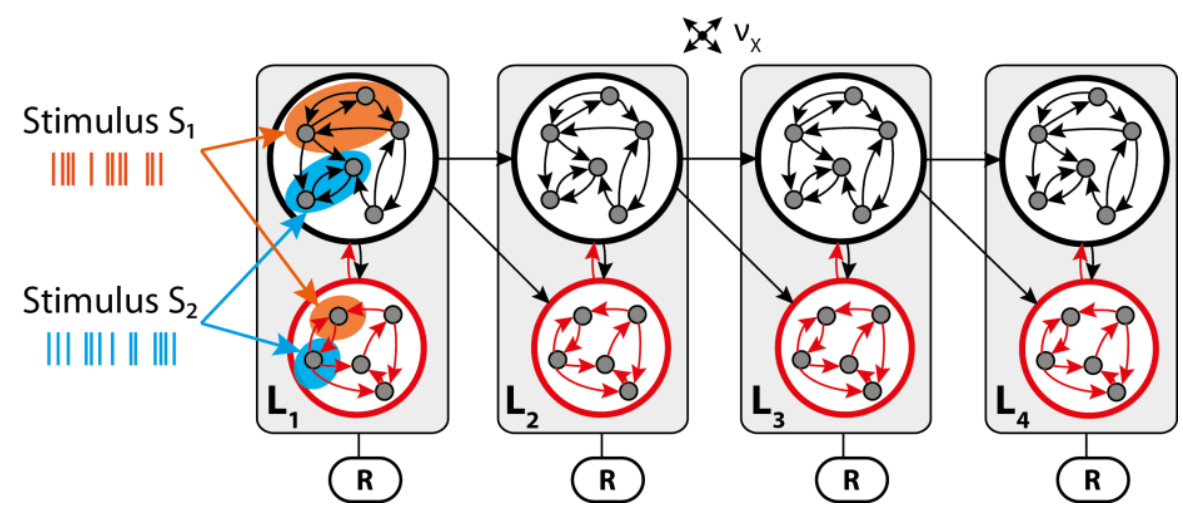

$>$ Each neuron $\rightarrow$ same amount of excitatory input

$>$ Tune parameters for asynchronous irregular activity (first 2 layers)

$>$ Readout from E populations in each layer

\section{Stimulus $\mathrm{S}_{2}$} ||| ||| || ||||

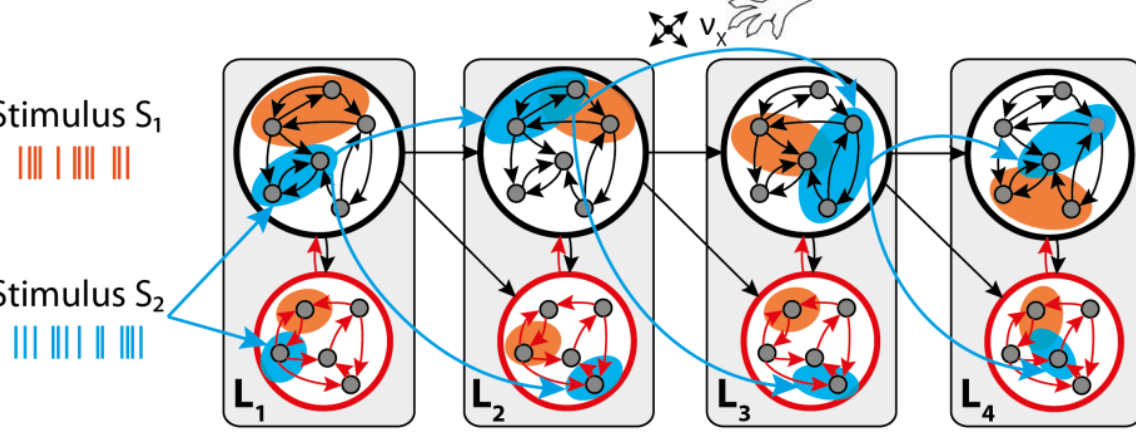

\section{topographic}

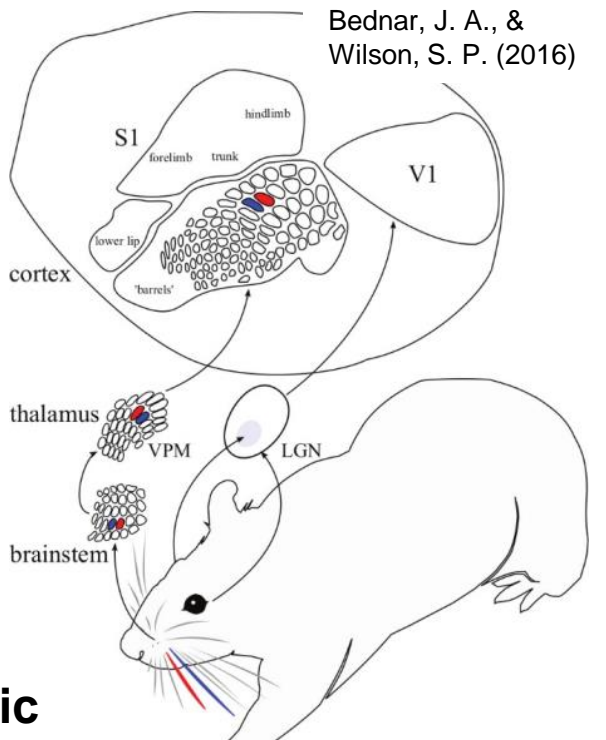

$>$ Stimulus-specific sub-populations conserved across the hierarchy through structured connectivity

$>$ Randomly selected, overlap allowed

$>$ Receptive field size fixed across the hierarchy

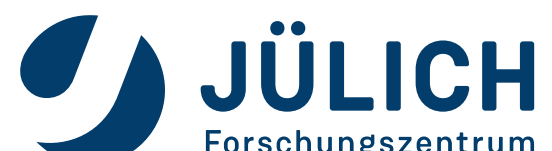




\section{Neural Microcircuit Simulation and Analysis Toolkit}

Python package to build, simulate and analyze complex neuronal microcircuits scalable and reproducible

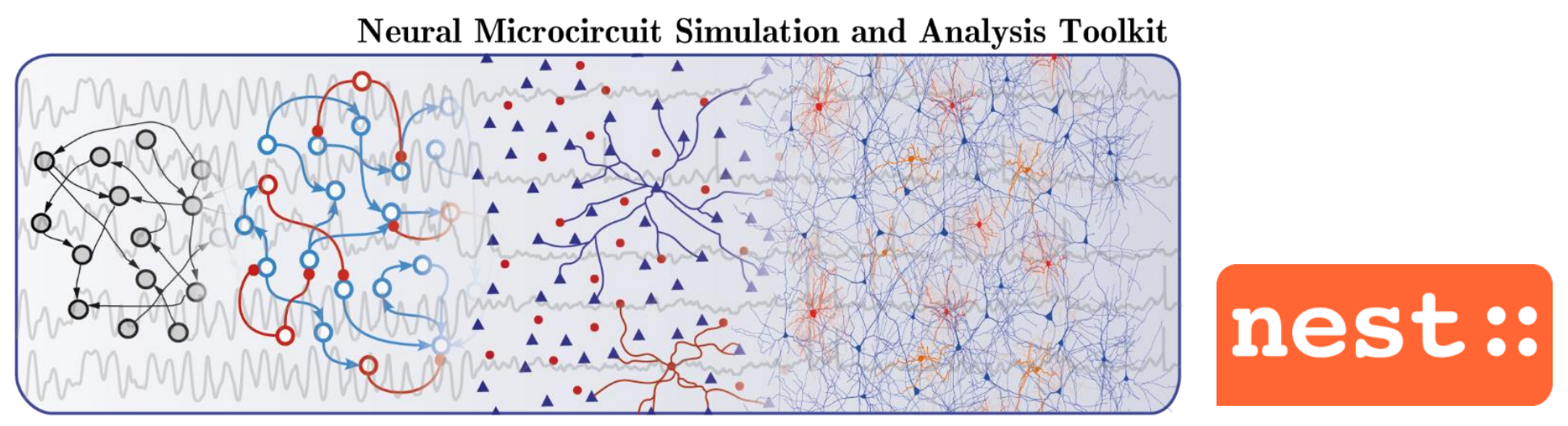

\section{GitHub}

https://github.com/rcfduarte/nmsat

\section{DOI $10.5281 /$ zenodo. 582645}

Renato Duarte, Barna Zajzon, \& Abigail Morrison. (2017). Neural Microcircuit Simulation and Analysis Toolkit

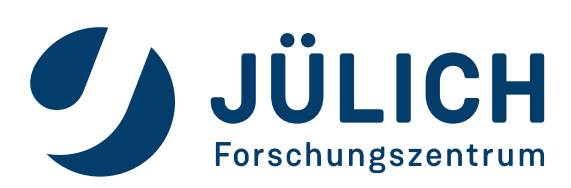




\section{Stimulus representation}
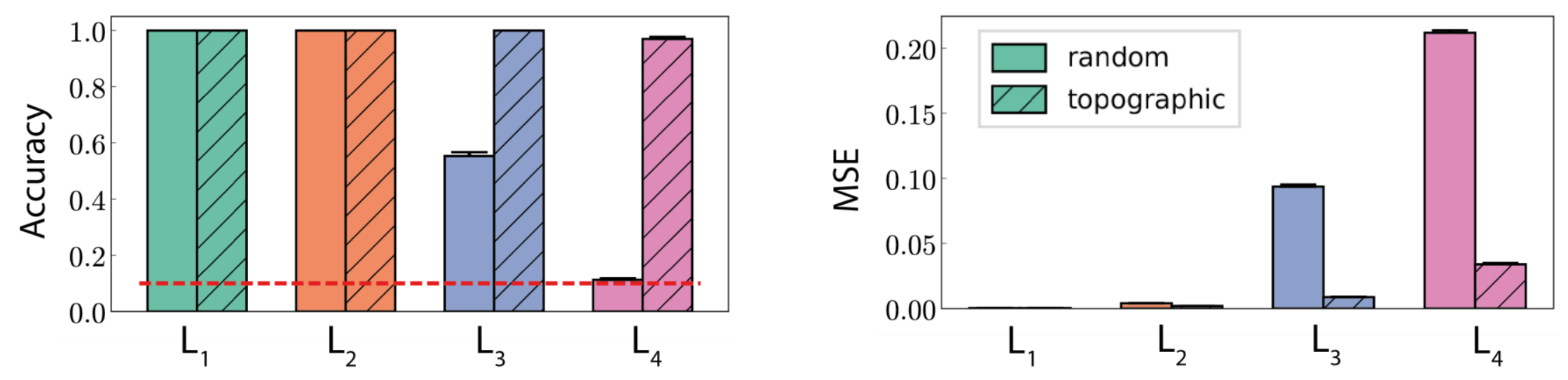

Classification of stimulus identity with $v_{\text {stim }}=15 \mathrm{spk} / \mathrm{sec}, 10$ stimuli

- Random connectivity enables stimulus decoding only up to $L_{3}$

- Topography improves accuracy in last 2 layers 


\section{Stimulus representation}

\section{Random network}

- Propagation depends on input rate

- No effect on $\mathrm{L}_{4}$

\section{Topographic maps}

- Capacity depends on connection density within topographic projections

- Compensates for increased overlap in case of more stimuli
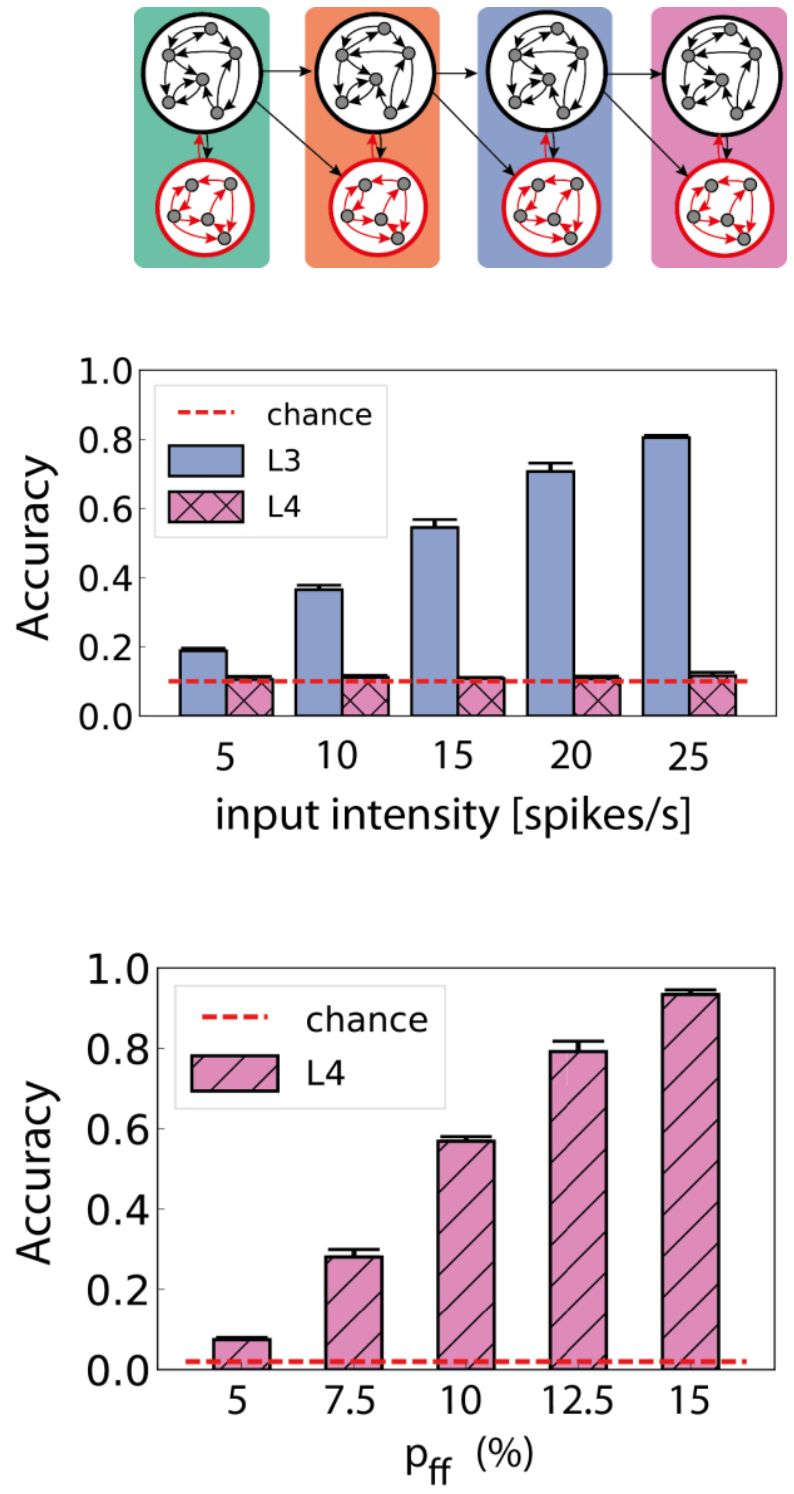

J 


\section{Activity statistics}
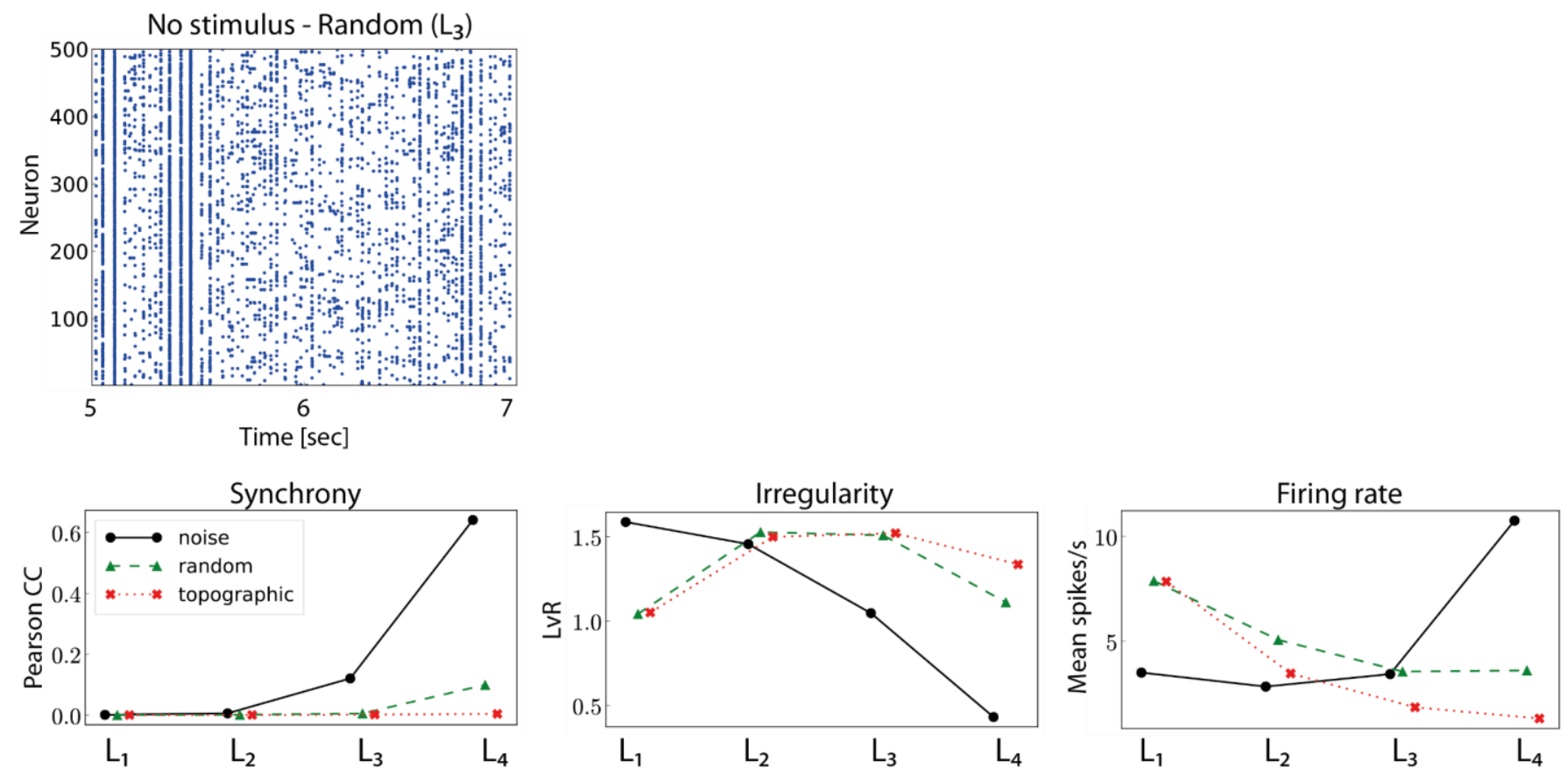

Noise-driven, no stimulus

- Firing is more regular

- Synchrony increases downstream (shared input effect) 


\section{Activity statistics}
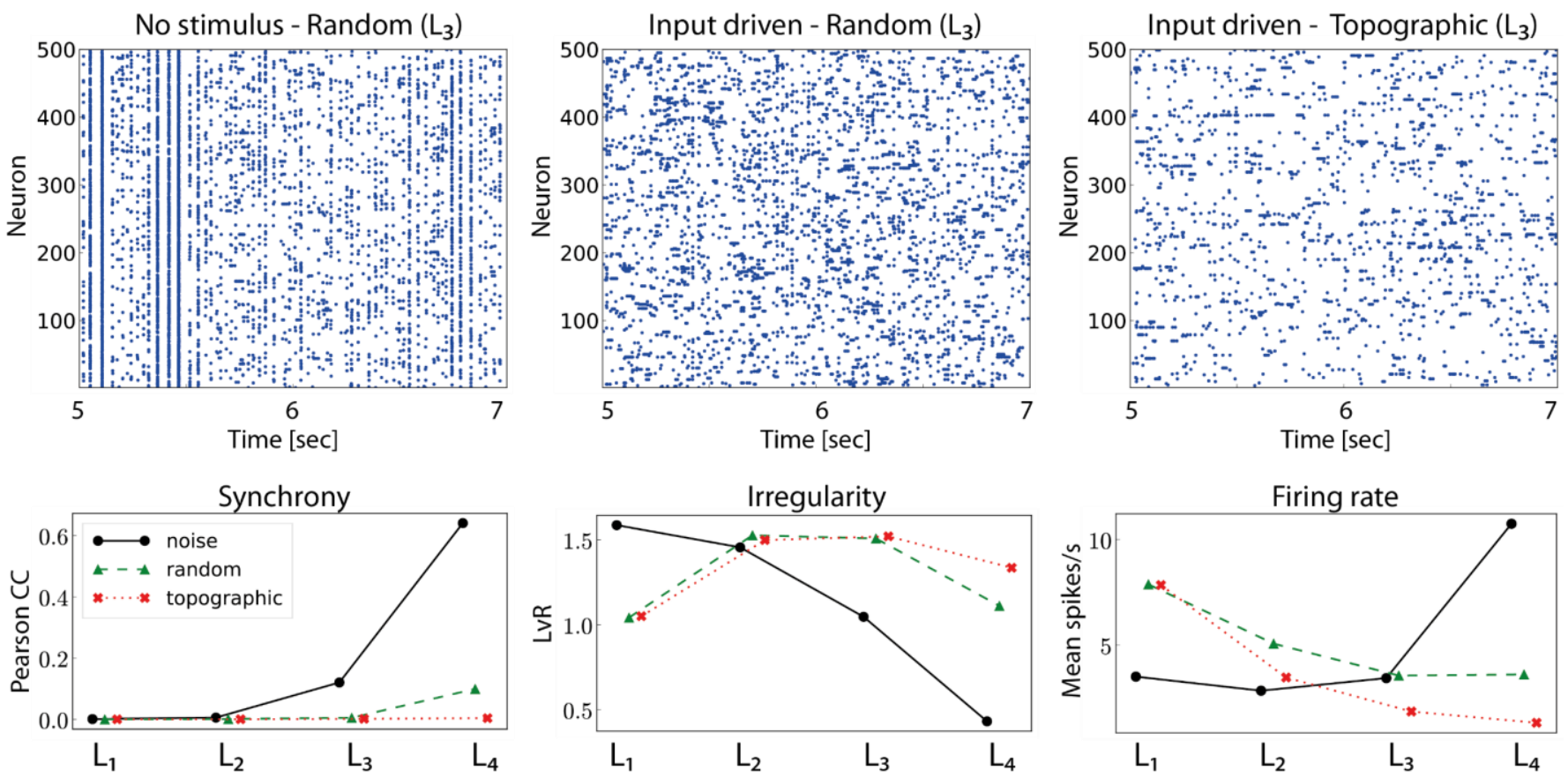

Noise-driven, no stimulus

- Firing is more regular

- Synchrony increases downstream (shared input effect)
Input-driven

- Global Al state maintained

- Synchrony reduced (topographic)

- Increased irregularity except $L_{1}$

Topographic networks are more resource-efficient 


\section{State space organization}

\section{Ideally $\rightarrow$ clearly segregated stimulus-specific clusters}
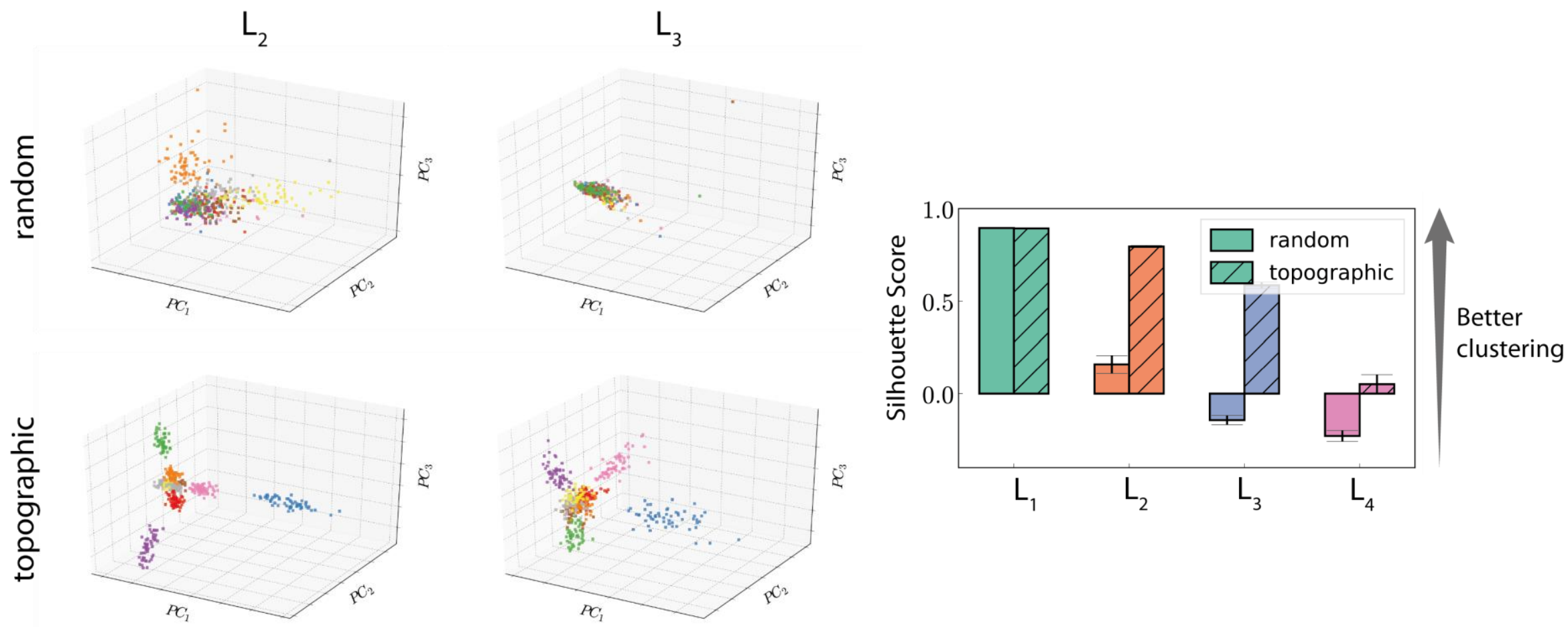

- Clustering quality decays through the hierarchy

- Topography leads to better state separation (silhouette score)

- In line with computational performance 


\section{State space organization}

\section{How are the networks exploring the high-dimensional state space?}

- Stimulus "enslaves" neural activity

- Effective dimensionality ${ }^{1}$ increases with hierarchical depth

- Topography extends duration of
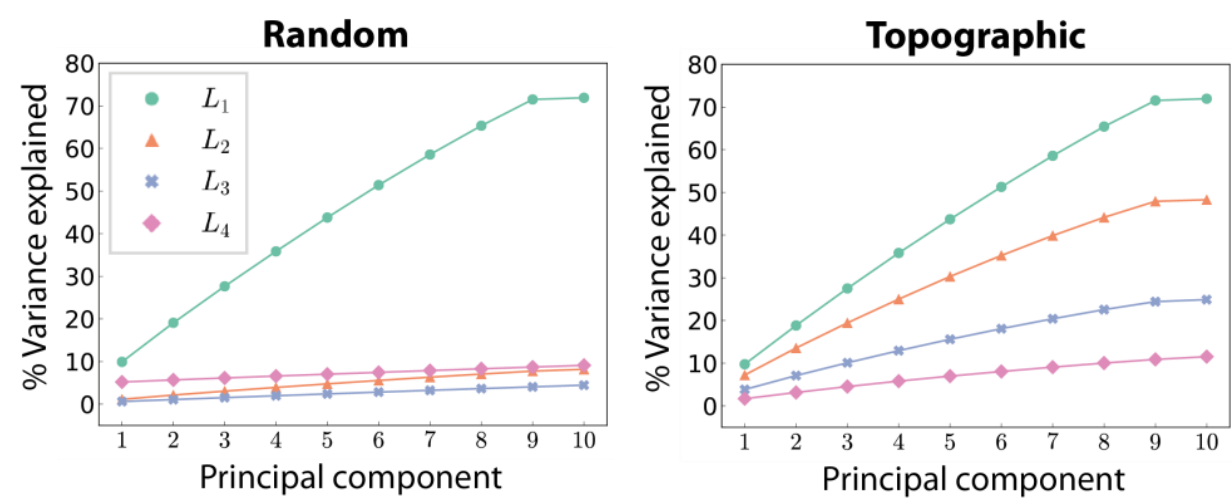
stimulus representations

- Dimensionality and variability inversely related to performance
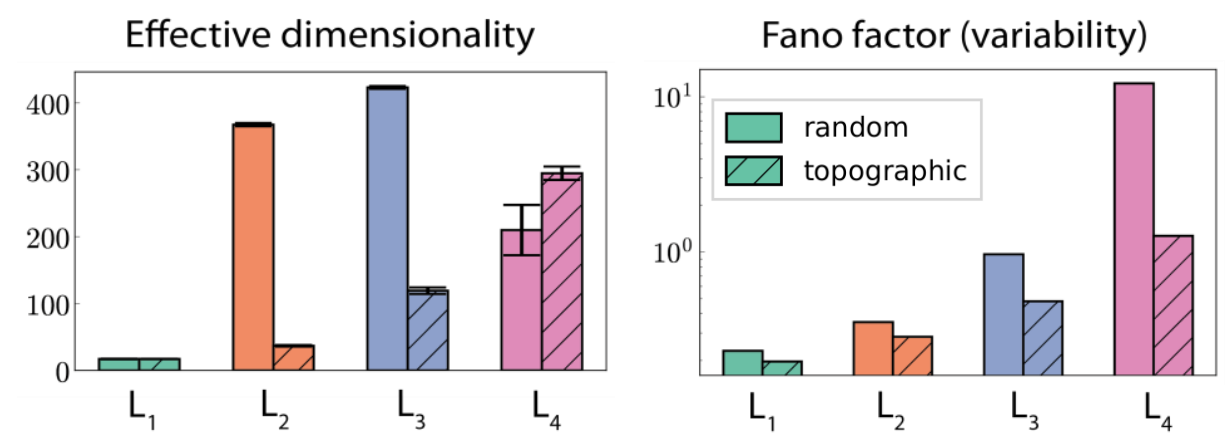


\section{Memory capacity and stimulus sensitivity}

\section{Stimuli presented sequentially}
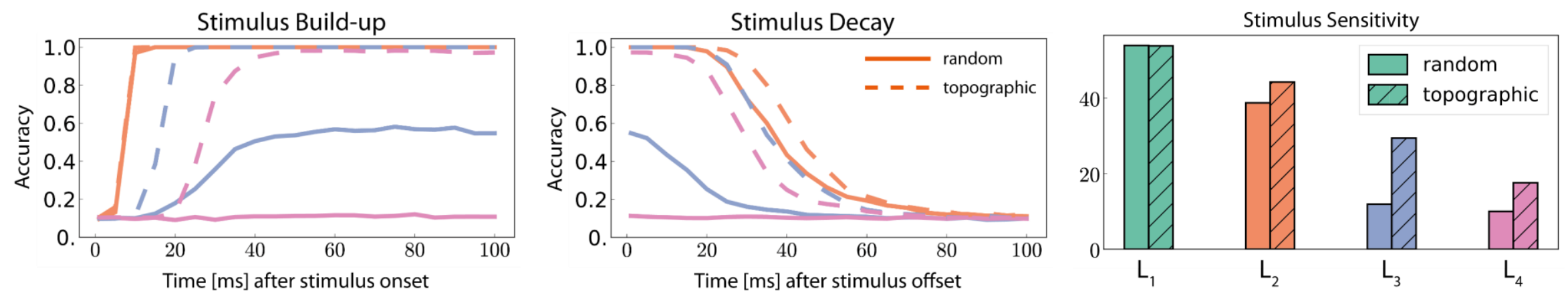

- Representations build-up over exposure time, slower downstream

- Topographic networks react faster

- Input representations gradually disappear (fading memory)

- Memory capacity decays with hierarchical depth

- Topography (marginally) increases memory

- Stimulus sensitivity: how long non-interfering representations are maintained $>$ decreases through hierarchy, better with topography 


\section{Summary}

Useful constraints for building hierarchical (balanced) spiking networks

- Random connectivity enough for local transmission, longer distances require topographic precision

- Topographic maps

(1) better performance

(1) memory capacity

(1) more efficient
( low-dimensional responses

(-) less variability

(2) more robust 


\section{Thank you!}

Member of the Helmholtz Association 